Presidential address 2009

\title{
Evidence based practice of the art of obstetrics
}

\author{
Malik Goonewardene ${ }^{1}$ \\ Sri Lanka Journal of Obstetrics and Gynaecology 2009; 31: 3-9
}

\section{The art of obstetrics}

According to the Holy Bible, on being tempted by the serpent, Eve plucked a fruit from the forbidden tree of knowledge, ate it and shared it with Adam. The pain of childbirth is said to be God's punishment for this initial sin of Eve.

Over the years, various measures have been adopted to relieve a woman's pain during childbirth and also to assist her to deliver her baby. The standing, semi erect, seated and squatting positions have all been adopted. The woman who assisted the mother and received the baby that was spontaneously delivered by the mother was referred to as the Midwife which means 'With Woman'. The famous Greek philosopher Socrates' mother is said to have been a midwife. Midwifery was a family profession which was handed down from mother to daughter and thus the traditional birth attendants came in to being. During these ancient times the husband too was involved in the process and gave comfort and emotional support to his wife.

Examples of easy labour, twin pregnancy with hand prolapse of the first twin and prolonged/ obstructed labour leading to a live birth but a maternal death are recorded in the Holy Bible. One of the earliest books of midwifery on record is the book "Byrth of Mankynd" written by Thomas Raynald in 1540. Up to the 19th century (the pre professional period) midwifery progressed slowly and moderate advances in the field were seen mainly in France. The pioneering maternity hospitals to be established in the world were the Queen Charlotte's Hospital in London (1752) and the Hotel Dieu in Paris (1788) . In the United Kingdom and Europe there was a symbiotic relationship between the female Midwives who attended on normal births and the male Barber-Surgeons and Obstetricians who attended on abnormal births. However, the midwives decided when the Barber-Surgeons or Obstetricians should be called in to attend on women

\footnotetext{
${ }^{1}$ President, Sri Lanka College of Obstetricians and Gynaecologists (2009),

Senior Professor and Head, Department of Obstetrics and Gynaecology, University of Ruhuna, Galle.

E-mail:malikg@eureka.lk
}

during childbirth. Therefore during this period, a midwife was in complete control of a woman during childbirth.

The latter part of the 19th century saw the transition from the pre professional period to the professional period, and the male Medical Obstetric Specialist was well established by the end of the 19th century. This 'medicalization of pregnancy and childbirth was highly criticized and objected to by the traditional midwives. Pharmacological and technological innovations were considered detrimental to a woman's experience and her family's freedom during a critical period of her life, which was a physiological phenomenon and usually not a medical problem.

\section{Position of the woman for normal child birth}

The Obstetric Specialists preferred the woman to be in the dorsal (recumbent) position for a normal child birth. Logistically this was the most convenient for the Obstetric Specialists. In his book 'Labour Among Primitive People' (1883), C. S. Englemann states:

"Uncivilized, savage people of the nations of the past (Red Indians) avoided the recommended dorsal position during child birth. They preferred the squatting position. Thinking Obstetricians will soon confirm the opinion of these ignorant but observing savages that the recumbent position retards labour and hinders easy, safe and rapid delivery".

However, the squatting position remains unpopular up to date is still not favoured and the dorsal or a semi recumbent position is preferred, for the convenience of the intra partum care giver.

\section{Prophylactic episiotomy and prophylactic forceps delivery}

The early part of the 20th century was the period of prophylaxis. Prophylactic episiotomy and prophylactic forceps delivery were advised by Prof. Joseph B de Lee (1869-1942), who stated that:

"parturition viewed with modern eyes is no longer a normal function. It has imposing pathological dignity". 
Charles B Reed another strong advocate of this philosophy stated:

"Medical practice is tending ideally towards prophylaxis. We strive to prevent pathology rather than await it onset".

Male Obstetric Specialists utilized technology to dominate and control childbirth. In his book 'Principles and Practice of Obstetrics' first edition (1913), de Lee however contradicts himself and states:

"The duty of the Accoucher is to observe the effects of nature, and not to aid until she has proven herself unequal to the task. Only when nature fails is art to enter. Nothing is so reprehensible as meddlesome midwifery".

In the fourth edition of his book (1924) he further states:

"Non interference of the process of nature and careful supervision ie watchful and armed expectancy has been proved to give best results".

It was during this period that Mary Breckenridge (circa 1930) rode on horseback and delivered more than 1000 babies in rural Kentucky, USA. She founded the Frontier Nursing Service in the Appalachian Mountains. Home deliveries were carried out and no maternal deaths were recorded, during a period when the maternal mortality rate in the hospitals in the region was approx. 50 per 1000 .

The 'monitoring period' commenced during the latter part of the 20th century. Childbirth was no longer divided in to 'normal' and 'abnormal'. There was a paradigm change in what was considered to be 'normal' and requiring 'normal management'. Child birth came to be divided in to physiological and psychological components with the 'normal' pole of childbirth being considered as the 'psychological component', which was handed over to the female midwife, the patient and her partner. The other pole was the 'physiological pole' and it was managed by the Obstetric Specialist (male or female) in order to optimize the outcome, and 'monitoring' became the key word. Although each member of the team had his or her own responsibility and became involved in this monitoring process, the Obstetric Specialist carried the overall responsibility.

\section{Monitoring child birth}

Fetal Heart Sounds (FHS) were first described in 1818 by Francois Mayor of Geneva. In 1882 Jean de Kergaradec published its significance. Pinnard from France described his well known Monoaural Fetoscope in 1880 . It was originally wooden and could be dismantled in to two pieces. Over the years aluminum and plastic versions have been designed and it is still being used worldwide, for intermittent auscultation of the FSH. With advances of fetal monitoring the Cardiotocograph (CTG) was designed and in certain centers women in labour are routinely monitored with a continuous CTG which greatly impairs their mobility. However, there is no scientific evidence to support this practice routinely in all women. In centers where intermittent auscultation (IA) and intermittent CTG is being carried out, changing over to continuous CTG monitoring is not recommended. Using the proper techniques, even the pathological late decelerations can be detected with IA. However, in the presence of tachycardia, IA should be used with caution because infrequent pathological variable decelerations could be missed.

\section{Forceps delivery}

The use of forceps to deliver a live child in England is credited to Peter Chamberlen the Elder. This 'secret instrument' was used exclusively by Peter Chamberlen and his male descendants for more than a century (circa 16th century) until the 'secret' was sold during the latter part of the 17th century.

However, several centuries earlier, the use of single bladed and later paired instruments have been mentioned in early Sanskrit writings (circa 1500 BC). These 'Jugna Sanker' have been used by ancient ayurvedic practitioners in India for 'difficult labour' with a dead fetus. There is evidence that these single and paired non articulated instruments have been subsequently used by Egyptians and then by Greeks and Arabs. Albucasis (AD 1013) is said to have developed and used short and long bladed obstetric forceps to deliver live babies.

Chamberlen's forceps were straight. The 'pelvic curve' was introduced independently by Leveret in Paris (1747) and William Smellie in London (1752) who also introduced the 'English Lock'. Axis traction devices were first introduced by Harmanne (1844) Tarnier (1877) and Milner Murray (1891) and subsequently modified by Neville Barnes and Hay Furguson who placed the axis traction rods on the handle and shank respectively. Piper introduced a forceps heavy unusually long and shanks a perineal curve, specially designed to deliver the after coming head of a breech presentation. Kjelland forceps (1951) and its subsequent modification by Moolgaokar (1962) has no pelvic curve and in this respect is similar to the original Chamberlen's forceps and has the distinct advantage of enabling rotation of the fetal head in the pelvis. It also has a sliding lock which enables the correction of asynctilism. Forceps delivery should be 
carried out by the correct person, on the correct patient, at the correct time, at the correct place, using the correct instrument. If not it can results in disaster to the baby, the mother, or both. The ability to carry out a forceps delivery remains a very important skill in the art of obstetrics and all obstetricians should be fully trained in forceps delivery.

\section{Vacuum extraction}

In 1694 James Yonge recorded a case of prolonged labour where a "cupping glass fix to the scalp with an air pump failed to draw out the head". In 1829 Niel Arnott advocated the use of "a pneumatic traction" which seems peculiarly adapted to a purpose of obstetric surgery. viz as a substitute for steel forceps in the hands of men who are deficient in manual dexterity, whether from inexperience or natural ineptitude"

James Young Simpsons is best remembered for his discovery of the anaesthetic properties of chloroform in 1847. In 1849 he demonstrated the effectiveness of his "Suction - Tractor; a new mechanical power as a substitute for forceps in tedious labour".

In 1953 Maalstrom described his vacuum extractor (ventous) where its suction had the unique feature of having its greatest diameter not at its point of application but at a level of $8 \mathrm{~mm}$ away from this point. This caused the scalp to be drawn in to the cup and become oedematous and thus imprisoned within the cup (the Chignon). Bird (1976) further modified this and developed the 'posterior cup' for occipito posterior position with separate attachments for suction and traction. The manual 'Kiwi Cup' vacuum extraction has been introduced recently. The ability to carry out a Vacuum Delivery is an essential skill which all obstetricians should have.

\section{Shoulder dystocia}

Shoulder dystocia, when the symphysis pubis prevents the anterior shoulder of the baby from being delivered, is an obstetric emergency. Although various risk factor assessments have been described, none have been effective in predicting its outcome. Therefore a large number of these cases cannot be prevented and occur unexpectedly. A great deal of skill is needed to manage a shoulder dystocia which commonly leads to brachial plexus palsies but may even result in a neonatal death. All intrapartum care givers should be trained in the art of managing a shoulder dystocia.

\section{External cephalic version}

The problems associated with delivering a baby with a breach presentation were appreciated even by
Aristotle (384 - 322 BC) who said:

"change the figure and place the head so that it can present at birth".

This refers to external cephalic version (ECV) which was extensively practised up to the middle of the 20th century. In the 1970s ECV fell in to disrepute and was abandoned as it was considered useless. In the 1980s there was renewed interest and today it is recommended as routine at term. All obstetricians should be trained in this art.

\section{Assisted vaginal breech delivery}

Whether to deliver a breach presentation vaginally or abdominally has been debated for several years. A well conducted large randomized control trial conducted in USA in 1980 showed that there was no difference in perinatal outcome of a breech presentation at term irrespective of whether the delivery was vaginal or abdominal. Two decades later (in 2000) the Canadian Term Breech Trial (TBT) concluded that planned Caesarean Section (CS) was better for the baby. However, they could not show any benefit for these babies at two years of age compared to those delivered vaginally. Five years later, the TBT was highly criticized because most cases of neonatal death and morbidity in the trial could not be attributed to the mode of delivery, and it was suggested that its recommendation should be withdrawn. In any case the benefits of CS for a breech presentation are mainly seen in countries such as Canada, United States of America (USA), United Kingdom (UK), Australia etc. (industrialized countries) which have low perinatal mortality rates. In non industrialized countries where obstetricians are thought to have greater expertise and clinical skills in AVBD, this benefit of CS is less. It is estimated that approximately $9 \%$ of breach presentations will require AVBD due to mothers' choice, patient establishing labour before ECV or being the second of twins. Furthermore the same technique of an ABVD is required when delivering a breech at CS. Therefore it is essential that all intra partum care givers are fully trained in the art of AVBD.

\section{Intra umbilical vein oxytocin and manual removal of placentae}

Retained placentae is a significant cause of post partum haemorrhage which continues to be the leading cause of maternal mortality in several countries including Sri Lanka. Recently intra umbilical vein (IUV) oxytocin has been shown to be effective for the management of retained placentae. All intra partum care givers should be trained to carry out this relatively simple procedure. However, obstetricians should continue to be trained in the art of manual removal of 
placentae because IUV oxytocin may not be effective in all cases.

\section{Caesarean section}

The oldest description of an abdominal surgical delivery of a baby is said to be found in a cuneiform tablet dating back to circa 2000 BC in Mesopotamia (current Iran \& Iraq). Numa Pompilius, the second king of Rome (762-715 BC) issued a decree (Lex Regia), by which it was forbidden to bury a dead pregnant woman without cutting out her child. This was supposedly to enable the child's soul to have an independent existence in the next world or next birth. During the reign of Augustus Caesar it was known as "Lex Caesarea" and from which the term caesarean Section (CS) is thought to have originated. Although the Greek Gods Adonis and Bachus are supposed to have been born by abdominal surgery, and those born by CS are thought to have mystical proven and extra ordinary strength, it is extremely unlikely that Julius Caesar was born by CS because his mother was supposed to be alive when he invaded Briton, and the mortality rate of CS was approx.100\% during that time.

The indications for a CS have evolved for its original post mortem indication to the current indication of preventing morbidity and mortality in both the baby and the mother. However, even today CS is associated with serious complications in both the mother and the baby. Detailed analyses carried out even in highly industrial countries such as the UK and the USA have shown that a mother has a 5-6 times greater risk of mortality after CS compared to after safe vaginal delivery. A complication which is of particular concern is a placenta praevia accreta which is dreaded by obstetricians all over the world as it carries an extremely high risk of death of the mother. Because the risk of a repeat CS in a subsequent pregnancy is considerably high the first CS should be done after careful thought, and only when obstetrically indicated. Trial of vaginal birth after CS should be encouraged in properly selected patients.

A Gynaecologist would not carry out a sterilization in a 19 year old girl who is engaged to be married just because she request it as her choice because she does not wish to have any children after marriage. A chef in a restaurant will provide either fish (baked or curry) or chicken (deep fried or oven roasted) depending on the clients' culinary preferences. However, even the chef needs to explain to the client that the fish curry is spicy hot and the chicken is not tender, and it is only then that the client can decide whether to have fish or chicken or whether to have a spicy dish or not. Counselling a woman regarding the appropriate mode of delivery requires a greater discussion of the pros and cons of the different modes of delivery and the final decision should be rational and based on sound obstetric principles, taking in to consideration the woman's informed choice. Prophylactic mastectomy, oophorectomy, hysterectomy, gastrectomy, colectomy, orchidectomy, amputation of penis etc are not carried out simply because patients are worried about developing cancers. Therefore CS on demand cannot be justified. Obstetricians should not become merely technicians carrying out the wishes of patients and relations. Even Prof JB de Lee, the champion of prophylaxis during the early part of the 20th century stated:

"a patient should be treated as judgment dictates. Obstetricians should act as practitioners of medicine, instead of catering to the wishes of patients".

\section{Learning the art}

In order to embark on an Evidence Based Practice of the Art of Obstetric Practice, one has to first learn the art. The Sigalovada Sutta which guides the behaviour of lay Buddhists clearly describes how a pupil should respect and honour his teacher (by rising, by attending on him, by attentive learning, by personal services and by respectfully receiving instructions)

The Hippocratic oath includes the statement

"To reckon him who taught me this art equally dear to me as my parents, to share my substance with him, and relieve his necessity if required, to look upon his offspring in the same footing as my brothers".

Over the years this oath has been modified several times by the World Medical Association, and the current version states

"I will give to my teachers the respect and gratitude which is their due"

and this version has been adopted by the Sri Lanka Medical Council (SLMC). Every medical practitioner is expected to affirm this at the time of his /her registration with the SLMC.

In the University of Ruhuna, the oath taken by the new entrant medical students includes the following statement.

"I resolve to respect with all my heart at all times those who teach, guide and help me in my endeavour to become a good doctor".

Our school teachers helped us build a sound foundation of knowledge. Our undergraduate and postgraduate teachers, as well as our colleagues and friends helped us not only to gain knowledge but also to improve our technical skills and gain further 
experience. We are indebted to our parents who have taught and guided us right through our lives and our families who supported us during our training. Therefore it is our bounded duty to thank all of them and respect them because they have contributed in great measure to what ever status we have achieved in our professional lives today.

After gaining knowledge as an undergraduate and acquiring certain skills during internship training in a teaching hospital, it is beneficial for a doctor aspiring to become a specialist in the future, to obtain further experience by working in smaller peripheral hospitals, before embarking on specialist training. Otherwise he/she will have 'tunnel vision' and a very narrow perspective. Specialist training involves gaining a great deal of advanced knowledge and acquiring specialized skills. The completion of postgraduate training per se is inadequate learning any art including obstetrics requires a commitment to life long learning and Continuing Professional Development (CPD). Organizations such as the World Health Organization and the Association of Commonwealth Universities assist in the CPD of obstetricians and we should be thankful to these organizations as well. While being engaged in CPD it is necessary that one should find time for sports, social interaction, relaxation and enjoyment.

"All work and no play make Jack a dull boy".

\section{Evidence based medicine and its critical appraisal}

Evidence Based Medicine (EBM) requires the integration of best available evidence, individual clinical expertise and the available facilities, and patient choice. New converts to the philosophy of EBM are often very keen to apply the best available evidence to their clinical practice but they fail to realize that clinical expertise and patient choice are equally important.

Allopathic medical practice is based on the scientific principles of evaluation of evidence (history, clinical features and investigations), logical reasoning based on sound theoretical knowledge (diagnosis), and applying the most effective and appropriate therapy.

Although rapid advances in science and technology have facilitated accurate diagnoses, the ability to interview a patient and obtain all relevant data, examine the patient and elicit all physical signs, pattern recognition in patients, and experience continue to be important characteristics of a good diagnostician. Similarly, application of the most effective and appropriate therapy to a patient requires not only updated knowledge but also skills and experience in its application. To practice EBM a clinician needs to be an excellent interviewer, diagnostician and therapist.

In any sphere of life, the more experienced person will in most instances perform better and have better results than a novice. However, if a clinician limits his practice to his own experience and that of a relatively few teachers and colleagues with whom he has interacted, he is prone to have a very narrow perspective and his practice will soon be out of date, sub-optimal and inappropriate.

Although clinician may be highly skilled in handling the latest technological advancements in the speciality, he should be able to manage his patients satisfactorily with the facilities available to him in his place of work. Informed choice of the patient is important. However, as mentioned earlier with reference to CS on demand, doctors should not become merely technicians carrying out the wishes of patients and relations.

Although a systematic review and meta analysis of several Randomized Controlled Trials (RCT) take pride of place as Level I evidence, sometimes a RCT may not be possible because it is unethical or not feasible. Furthermore both primary as well as secondary research may be done badly, can have limitations, and be biased. External Best Evidence may sometimes be generated by non clinicians and statisticians trained in the process of Systematic Reviews and Meta Analyses. Results and conclusion of these systematic reviews may be invalid in real life and sometimes the conclusions may in fact be wrong. Therefore Clinicians should not be like parrots blindly following the recommendations of these reviews. The WHO Reproductive Health Library is a good source of external based medicine.

External best evidence is also not always black and white. Clinicians are constantly dealing with the infinite variables of patient presentation and their response to treatment, and 'grey areas' when they manage individual patients. Therefore the clinician has to blend and balance the art of uncertainty verses the science of probability. In the presence of sound scientific evidence the clinician is expected to act according to this evidence.

Best available evidence gives an average effect for an average patient It does not consider severity of symptoms, severity of disease, co-morbidity and individual idiosyncrasies. It also does not help clinical decisions based on pathophysiological psychosocial and economic issues. Therefore a 
summary statistic derived from a meta analysis of a systematic review will not be the best for every patient. In RCT and Meta Analyses it is necessary to decide to whom these results apply.

Best available evidence also changes over time. Several clinical procedures which have been used in the management of pregnancy and child birth for several years in the past have been subsequently condemned as being ineffective or inappropriate and therefore discarded. As a result of the natural cycle of change and/or EBM some of these procedures are regaining popularity and are being reintroduced now. Examples of such procedures include external cephalic version, the use of intra cervical catheters for preinduction cervical ripening, vaginal birth after CS, artificial separation of membranes, and positioning of the neonate.

The ability to critically appraise research publications in terms of its quality, validity generalizability, applicability, feasibility and cost effectiveness to an individual patient is an important characteristic of a clinician. EBM can build on and reinforce clinical expertise. However, it can never replace clinical skills, judgment and experience. Clinical expertise and experience is essential to critically appraise and decide whether the external best evidence is appropriate to the individual patient at all, and if applicable how it should be applied. Common sense is also essential.

\section{Research and audit}

Research and audit are integral aspects in the practice of any discipline of curative or preventive medicine including obstetrics and gynaecology. Research answers the question 'what should be done?' and Audit answers the question 'Is it being done?' Research is not an entity confined to Science and Medicine. Human beings (even animals) carry out research in their day to day lives for their own existence. Whether it is for purchasing a shirt, trouser, sari or shoes, or for hosting friends for a meal at a restaurant we carry out research. We gather information from media (newspapers, television or radio) friends and relations and carry out a preliminary evaluation or use our previous experience. Then only would we select the best in terms of appearance, comfort, durability, reliability, feasibility, safety, cost effectiveness or taste.

This is research and it is an integral part of one's day to day life. Therefore, we should not be scared or reluctant to carry out clinical research. Very often the excuse is 'no time', 'no resources' or 'no funds'.
There are numerous areas for research available in Sri Lanka. A senior colleague has described very well the different ways that a researcher can select a topic and these are a) It is a national or local priority, b) It is your personal preference, c) It is your bosses preference, d) It is easy, e) It is prestigious, f) It can be funded, and g) It has the highest commercial return. The researcher has the freedom to select the topic. Some topics related to the art of obstetrics and relevant to an evidence based practice of the art in Sri Lanka, which need more research include:

1. Effective antenatal oral iron supplementation and reduction of iron deficiency anaemia in pregnancy.

2. The value of Customized Symphysio Fundal Height Charts

3. Estimation of birth weight centiles at different periods of gestation

4. Methods of pre induction cervical ripening: The use of Nitric Oxide Donors vaginally and the use of the Folly Catheter intra cervically

5. The value of the Fetal Acoustic Stimulation Test for fetal monitoring: antenatally as well as intrapartum

6. The value of amnioinfusion: for potential or suspected umbilical cord compression in labour and for meconium stained amniotic fluid

7. The most suitable position for a normal vaginal delivery

8. The value of routine Episiotomies

9. The place of Assisted Vaginal Breech Delivery

In the year 2009, the Sri Lanka College of Obstetricians and Gynaecologists (SLCOG) plans to continue the island wide Safe Motherhood Activities and Training Workshops which have been going on for many years. However a greater emphasis will be given to improving intrapartum care.

In addition the commencement of two additional programmes are envisaged to address:

- Reduction of maternal morbidity and mortality due to postpartum haemorrhage.

- Reduction of maternal morbidity and mortality due to Unsafe Abortion.

My vision for the future is that all the fellows and members of the SLCOG would make a firm commitment to:

1. Abide by the moral and ethical codes of conduct expected. 
2. Respect the teachers who taught this art.

3. Respect colleagues (juniors and seniors) from whom much more can be learnt about the art.

4. Become actively involved in Continuous Professional Development on a life time learning basis, and gather further knowledge and skills while gaining experience in the specialty.

5. Practice Evidence Based Medicine (EBM), while appreciating that results and conclusions from primary and secondary research could be flawed, may nor be generalizable or applicable to individual patients, that evidence changes over time and that adequate clinical acumen and skills are needed when adopting EBM for the management of individual patients.

6. Become actively involved in research and audit.

7. Improve intra partum care.

8. Reduce maternal morbidity and mortality due to post partum haemorrhage.

9. Reduce maternal morbidity and mortality due to unsafe abortion. 